\title{
Observation of Resonant Energy Transfer Between Identical-Frequency Laser Beams
}

\author{
K. B. Wharton, R. K. Kirkwood, S. H. Glenzer, K. G. Estabrook, \\ B. B. Afeyan, B. I. Cohen, J. D. Moody, B. J. MacGowan, C. Joshi
}

This paper was prepared for submittal to the American Physical Society Division of Plasma Physics Meeting New Orleans, LA

November 16-20, 1998

December 9, 1998

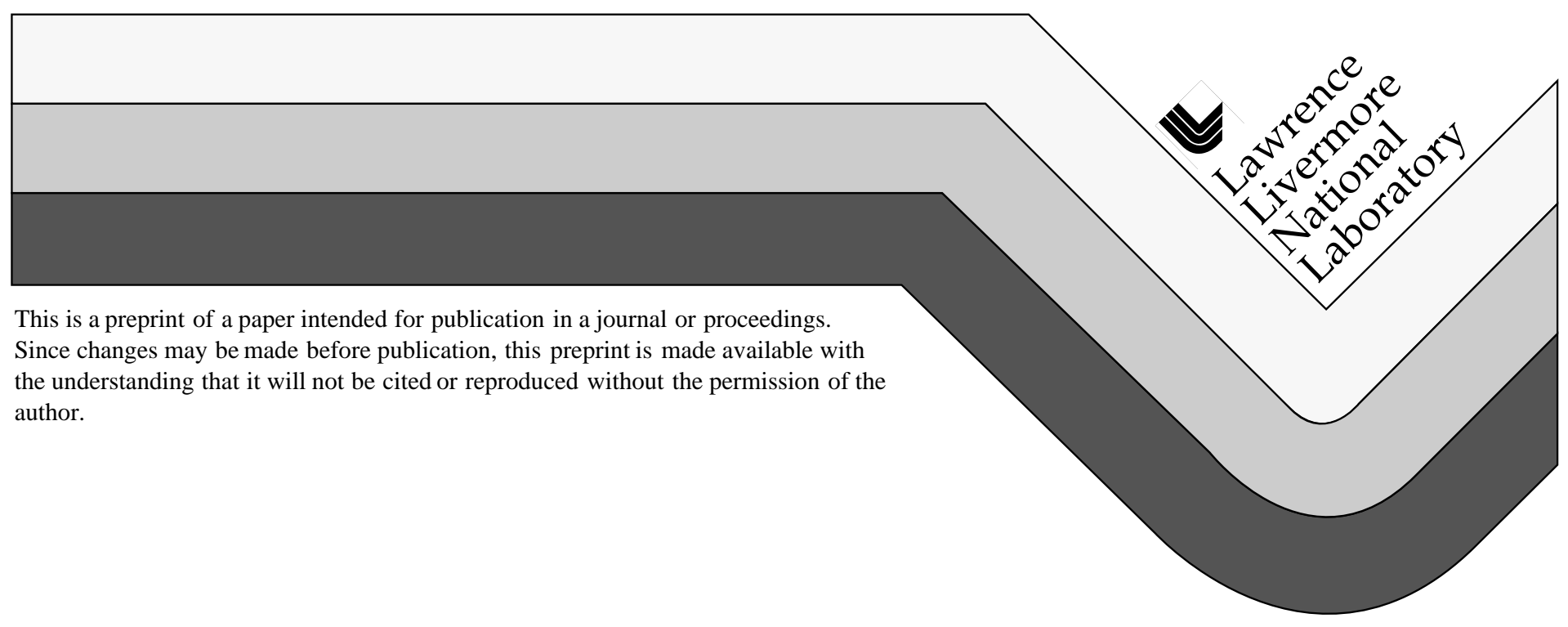




\section{DISCLAIMER}

This document was prepared as an account of work sponsored by an agency of the United States Government. Neither the United States Government nor the University of California nor any of their employees, makes any warranty, express or implied, or assumes any legal liability or responsibility for the accuracy, completeness, or usefulness of any information, apparatus, product, or process disclosed, or represents that its use would not infringe privately owned rights. Reference herein to any specific commercial product, process, or service by trade name, trademark, manufacturer, or otherwise, does not necessarily constitute or imply its endorsement, recommendation, or favoring by the United States Government or the University of California. The views and opinions of authors expressed herein do not necessarily state or reflect those of the United States Government or the University of California, and shall not be used for advertising or product endorsement purposes. 


\title{
Observation of Resonant Energy Transfer Between Identical-Frequency Laser Beams
}

\author{
K.B. Wharton1,2, R.K. Kirkwood2, S.H. Glenzer2, \\ K.G. Estabrook2, B.B. Afeyan3, B.I. Cohen2, \\ J.D. Moody' , B.J. MacGowan ${ }^{2}$, and C. Joshi1
}

1) University of California, Los Angeles, California 90024

2) Lawrence Livermore National Laboratory, University of California, P.O. Box 808, Livermore, California 94550

3) Polymath Associates, Livermore California 94550 and University of Nevada, Reno, Nevada 89557

Enhanced transmission of a low intensity laser beam is observed when crossed with an identical-frequency beam in a plasma with a flow velocity near the ion sound speed. The time history of the enhancement and the dependence on the flow velocity strongly suggest that this is due to energy transfer between the beams via a resonant ion wave with zero frequency in the laboratory frame. The maximum energy transfer has been observed when the beams cross in a region with Mach 1 flow. The addition of frequency modulation on the crossing beams is seen to reduce the energy transfer by a factor of two. Implications for indirect-drive fusion schemes are discussed.

PACS numbers: 52.40.Nk, 42.65.Ky, 52.35. Nx 


\section{Wharton $/ 2$}

I. INTRODUCTION

Energy transfer between two intersecting laser beams in a plasma directly addresses fundamental aspects of laserplasma interactions and is also relevant to laser-driven inertial confinement fusion. Current designs for fusion experiments on the National Ignition Facility (NIF) [1] have multiple laser beams crossing as they enter a cylindrical radiation enclosure (hohlraum). Energy exchange between the laser beams would have deleterious effects on the symmetry of the laser radiation inside the hohlraum, and could require the use of NIF's ability to frequency detune the crossed beams and avoid a resonance.

When an electromagnetic wave (frequency $\omega_{\circ}$, wavenumber $\mathbf{k}_{\mathbf{0}}$ ) intersects another electromagnetic wave with equal or lower frequency $\left(\omega_{1}, \mathbf{k}_{1}\right)$, optical mixing will drive a beatwave density perturbation in the plasma at $(\Omega, \mathbf{K})$ :

$$
\Omega=\omega_{0}-\omega_{1} \quad ; \quad \mathbf{K}=\mathbf{k}_{\mathbf{0}}-\mathbf{k}_{\mathbf{1}}
$$

If the driven beat-wave is a normal mode of the plasma then this three-wave interaction is resonant and can be very efficient [2,3]. Electron plasma waves can be driven in this manner, using two laser beams with a difference in frequency equal to the electron plasma frequency $[2,4]$, and this technique has found applications in particle acceleration [57]. For beams of comparable frequency, resonance can be 
reached when $(\Omega, \mathbf{K})$ satisfies the dispersion relation $\left(\Omega=\omega_{i a}, \mathbf{K}=\mathbf{k}_{\mathbf{i a}}\right)$ for ion acoustic waves in a flowing plasma:

$$
\omega_{i a}=c_{s}\left|\mathbf{k}_{\mathbf{i a}}\right|+\mathbf{v}_{\mathbf{d}} \bullet \mathbf{k}_{\mathbf{i a}}
$$

Here $c_{S}$ is the ion sound speed and $\mathbf{v}_{\mathbf{d}}$ is the drift velocity of the plasma. In subsonic plasmas $\left(\left|\mathbf{v}_{\mathbf{d}}\right|<\mathrm{c}_{\mathrm{s}}\right), \omega_{\text {ia }}$ is non-zero, which with Eq.(1) shows that identical frequency beams cannot drive a resonant ion wave. With the appropriate frequency mismatch, however, resonant ion waves have been driven by microwaves [8] and also by two laser beams [9] . This latter experiment measured a modest transfer of energy mediated by a resonant ion wave, but no energy transfer was observed when the frequency mismatch was set to zero. Non-resonant ion waves have been produced with two identical frequency beams, which have been found to have an effect on Stimulated Raman Scattering [10]. Lal et al. have observed energy transfer between two $\lambda=10.6 \mu \mathrm{m}$ wavelength laser beams [11], but this was during a transient period on the order of a few acoustic periods, during which nonresonant energy transfer may occur $[12,13]$. These previous experiments were performed in subsonic plasmas.

In a supersonic plasma $\left(\left|\mathbf{v}_{\mathbf{d}}\right| \geq \mathrm{c}_{\mathbf{s}}\right)$ the resonant ion wave can have zero frequency in the laboratory frame $\left(\omega_{\text {ia }}=0\right)$, and the ion wave can therefore transfer energy between two identical frequency beams over many acoustic periods [14-16]. The spectral width of this resonance is roughly equal to the 


\section{Wharton/4}

damping rate of the ion wave, $\operatorname{Im}\left(\omega_{\text {ia }}\right)$, as seen by the dependence of the two-beam frequency separation $\Omega$ in the gain exponent Q for a homogeneous plasma [17]:

$$
Q \propto \frac{1}{1+4\left[\left(\Omega-\omega_{i a}\right) / \operatorname{Im}\left(\omega_{i a}\right)\right]^{2}}
$$

The resonance width can be further increased by adding spectral bandwidth to the crossing beams. Resonant energy transfer via a zero-frequency ion wave has been the subject of much theoretical work $[13,17-19]$, and has recently been experimentally demonstrated for the first time [20]. Results from these previous experiments will be summarized below, along with new experimental results from beams with added frequency modulation.

\section{EXPERIMENT}

These experiments were performed on the 10-Beam Nova laser facility at Lawrence Livermore National Laboratory, using four f/4.3 beams with $\lambda=351 \mathrm{~nm}$. Two of these beams were defocused and used as "heaters" to explode a thin foil; the other two interaction beams were then crossed in the resulting plasma. The higher intensity interaction beam is called the "pump", while the lower intensity interaction beam

is the "probe"; these two beams have a potential for resonant energy transfer.

The heaters were partially defocused to $800 \mu \mathrm{m}$ diameter spots, each spatially smoothed with a kinoform phase plate (KPP) [21] and containing $3 \mathrm{~kJ}$ of energy in a square pulse 


\section{Wharton $/ 5$}

lasting for 3ns. These two beams were incident $(400$ to normal) on both sides of a $5 \mu \mathrm{m}$ thick $\mathrm{Be}(\mathrm{Z}=4)$ rectangular foil, 2 by $4 \mathrm{~mm}$ in size. LASNEX [22] modeling indicated a layer with Mach 1 flow $\left(\left|\mathbf{v}_{\mathbf{d}}\right|=c_{\mathbf{S}}\right)$ moved out from the initial foil position over time, reaching a distance of $500 \mu \mathrm{m}$ from the foil at t=3ns. At this time the density along the center normal of the foil had reached a 1mm-scale plateau of a roughly constant electron density $\mathrm{n}_{\mathrm{e}}=0.06 \mathrm{n}_{\mathrm{C}}$, where $\mathrm{n}_{\mathrm{C}}$ is the critical density for $351 \mathrm{~nm}$ light (9 $10^{21} \mathrm{~cm}^{-3}$ ).

The flow velocity was experimentally characterized with a Thomson scattering technique $[20,23]$. A lower intensity $\lambda$ $=526 \mathrm{~nm}$ beam was focused in a $100 \mu \mathrm{m}$ FWHM spot, $500 \mu \mathrm{m}$ from the center foil position. The frequency- and time-resolved Thomson scattered light is shown in Figure 1a. At $t=2.9 n s$ the up-shifted ion wave feature overlaps the stray light, signifying that $\omega_{i a}=0$ and that a Mach 1 plasma flow was present. Figure 1b shows that this measured Mach 1 position $(z=500 \mu m, t=2.9 n s)$ is consistent with the LASNEX calculation. However, the measured electron plasma temperature at $t=2.9 \mathrm{~ns}$ is $0.8 \pm 0.1 \mathrm{keV}$, lower than the predicted $1.2 \mathrm{keV}$ at this location. The error bars of the Mach 1 measurement are largest in the direction away from the foil due to the possibility that the beam was deflected by the plasma density gradient, with a maximum error defined by the spatial view of our diagnostic. Even with this effect, the error in the Mach 1 measurement is much smaller than the spatial and temporal 


\section{Wharton/6}

extent of the region sampled in the main experiment, as shown by the bashed box in Figure 1 b.

The geometry of the crossed-beam interaction is shown in Figure 2. The pump and probe beams arrived from opposite directions, separated by an angle of $152^{\circ}$. Both the pump and probe were incident at $14^{\circ}$ from the normal of the foil (the z-axis, defined in Figure 2), and the resultant ion wave was therefore aligned to the plasma flow along the z-axis. The pump and probe beams were originally focused at a location $z=-500 \mu m$ from the $z=0$ initial foil position. The pump has a higher frequency than the probe in the frame of the flowing plasma on the $-z$ half of the foil, and therefore a resonance there would be expected to transfer energy from the pump to the probe, rather than in the opposite direction.

The probe light transmitted through the plasma was measured on each shot $[20,24]$, giving a temporally resolved (100ps resolution) transmission percentage for the probe beam. A similar diagnostic allowed for transmission measurements of the pump beam.

All of the experiments used a pump beam with $3 \mathrm{~ns}$ duration and smoothed with a $\mathrm{KPP}$, yielding a $10^{15} \mathrm{~W} \mathrm{~cm}-2$ peak intensity over a $340 \mu \mathrm{m}$ diameter spot. The probe beam had a typical energy of 0.2 to $0.3 \mathrm{~kJ}$, and two focal spots were used. For the first experiments, no phase plate was used on the probe, allowing a focused $100 \mu \mathrm{m}$ spot (full-width at halfmaximum; $170 \mu \mathrm{m}$ full diameter). The probe's pulse shape was a 
3ns upward ramp, beginning at $t=1$ ns and reaching a peak of $150 \mathrm{GW}$ at $\mathrm{t}=4 \mathrm{~ns}$.

\section{TRANSMISSION MEASUREMENTS}

The transmission of the probe beam described in Sec. II is plotted against time in Figure 3a. With no pump beam present, the transmission of the probe through the exploding foil stabilized at 50-60\%; a partial absoprtion due to inverse bremsstrahlung in the plasma. With the addition of the pump beam, crossing the probe at a location $z=-500 \mu m$, the transmission of the probe increased to near $100 \%$ on short timescales. However, when the two beams were crossed at a location $z=-750 \mu m$, the probe transmission returned to the previous 50-60\% level. Because the probe passed through the bulk of the exploding foil plasma before reaching the crossing region, this region had an average intensity ratio $I_{\text {pump }} / I_{\text {probe }}$ of $\sim 3$.

To change this intensity ratio, a kinoform phase plate was then added to the probe beam, increasing the spot size to $340 \mu \mathrm{m}$ (full diameter). Also, the probe's pulse shape was changed to a $4 \mathrm{~ns}$ square pulse, extending from $t=1 \mathrm{~ns}$ to $t=5 \mathrm{~ns}$, raising the average $I_{\text {pump }} / I_{\text {probe }}$ to $\sim 25$.

Figure $3 b$ shows the transmission of this lower-intensity probe beam, both with and without the pump beam. The no-pump transmission was nearly identical to the previous case. With the pump beam present, the transmission was again increased to $100 \%$ levels when the beams were crossed at $z=-500 \mu m$; 


\section{Wharton/8}

40\% levels when the beams were crossed at the original foil position $(z=0)$; and no significant transmission enhancement when the beams were crossed at $z=+500 \mu m$, or the "Mach -1 " position.

The crossed-beam experiments at $I_{\text {pump }} / I_{\text {probe }}=25$ have recently been extended to beams with $1 \AA$ of frequency modulation (peak-to-peak at $351 \mathrm{~nm}$ ). Both the pump and probe beam were modulated at $17 \mathrm{GHz}$, so that the laser wavelength fluctuated by $1 \AA$ over a 59ps full-cycle. Neither beam was spectrally dispersed. The relative phase between the pump and the probe beams at the interaction point was unknown, but the two beams were unlikely to be in phase from purely statistical considerations.

Transmission of this spectrally modulated probe beam is shown in Figure 4. The spikes in the transmission data (as compared to Figure 3) are due to the frequency modulation of the incident beam. Again, the no-pump transmission was 50$60 \%$, but the probe transmission with the pump present (crossed at $z=-500 \mu \mathrm{m}$ ) was $70-80 \%$. This is significantly lower than the corresponding 90-100\% transmission seen without modulation. 
Wharton/9

IV. ANALYSIS

The varying transmission fractions of the probe beam in the above experiments suggests that energy may be resonantly transferred from the pump to the probe. It is assumed that the two "heater" beams do not exchange energy with the probe, because of the lower intensity of the defocused heater beams as well as an unoptimized geometry with respect to the plasma flow. Even if this assumption is incorrect, the heater beams are present on every shot, so any heater beam resonance would be irrelevant for a pump-on/pump-off comparison.

However, increased transmission of the probe beam in the presence of a pump is not by itself convincing evidence of energy transfer. Plasma heating by the pump beam might decrease the inverse bremsstrahlung absorption of the probe beam, giving the appearance of energy transfer. LASNEX simulations show no pump beam effect on the plasma density or Mach 1 location, but do show a higher temperature plasma when the pump is on. This temperature change increases the peak theoretical probe transmission from $60 \%$ to $70 \%$, but cannot explain the observed $\sim 100 \%$ transmission. In addition, this effect is calculated to occur late in the pump pulse, from $\mathrm{t}=3 \mathrm{~ns}$ onward (after maximum pump heating), rather than the earlier $t=\sim 2$ ns where our peak transmission is observed. Further evidence against this pump-heating scenario includes a measurement of the transmission of the pump beam; it peaks later in time $(t=3.2 \mathrm{~ns})$, at a level of 55\%, while the amplified probe signal peaks earlier $(t=2.4 \mathrm{~ns})$ and with much 
higher transmission levels. Because the two beams are not exactly collinear, any pump-induced heating should have primarily increased the pump transmission; the opposite is observed.

For each transmission measurement, the factor Gprobe by which the probe beam was enhanced can be computed by simply dividing the crossed-beam transmission by the no-pump transmission. Although the early-time peak enhancements are large ( $\left.G_{\text {probe }}>3\right)$ in several cases, the corresponding errors are large as well because of the lower no-pump transmission values at these times. A more quantitative measurement can be made by averaging $G_{\text {probe }}$ over $2 \mathrm{~ns}<\mathrm{t}<3 \mathrm{~ns}$, the time period when the Mach 1 flow velocity is calculated to be between $z=-300 \mu m$ and $z=-500 \mu m$. These averages are plotted vs. position in Figure 5. The large horizontal error bars represent the extent in the z-axis of the high-intensity diamond-shaped crossing region of the two beams. The maximum Gprobe of $\sim 1.6$ occurred when the crossing region overlapped the Mach 1 region; little enhancement was observed when the beams were crossed outside this region. This dependence on position is strong evidence of a resonant process.

One striking observation from Figure 5 is that the frequency modulation on the crossing beams seems to lower the amount of energy transfer. The depth of the modulation was probably not sufficient to detune the resonance out of the crossing region; there should have still been an ion wave resonance at all times. Instead, the observed effect might 
be due to a modulation of the resonant flow velocity corresponding to the beam modulation. If a $180^{\circ}$ relative phase existed between the $17 \mathrm{GHz}$ modulation on the Pump and Probe beams, the resonant flow velocity would have been shifted from Mach 1.2 to Mach 0.8 in the span of 30ps. Plasma in the beam-crossing region with a given flow velocity (say Mach 1.0) would have been resonant only for 10 ps each half-cycle. Smaller phase-shifts between the pump and probe beams would result in correspondingly longer resonance times. Still, these times are comparable to the inverse of the ion wave damping frequency (7.5ps). BZOHAR [25], a twodimensional electromagnetic code that uses particle ions and Boltzmann fluid electrons, shows early ion-wave saturation on a 20ps timescale [26]. The relative timescales and the experimental results indicate it is plausible that the modulation limited the growth of the resonant ion-wave.

Another interesting aspect of Figure 5 is that only $\sim 10 \%$ of the pump energy is transferred to the probe. This is in contrast with the near-100\% transfer predicted by assuming a linearly driven ion wave in a homogenous plasma, where the exponential gain is given (within a near-unity factor) by [17]:

$$
Q \cong\left(\frac{\omega_{p e}}{\omega_{0}}\right)^{2}\left(\frac{\mathrm{v}_{\text {osc }}}{\mathrm{v}_{\mathrm{e}}}\right)^{2} \frac{k_{i a} c_{s}}{\operatorname{Im}\left(\omega_{i a}\right)} \frac{L}{\lambda_{0}}
$$

The term from Eqn. (3) is neglected, as it is unity at exact resonance. Here $\omega_{\text {pe }}$ is the plasma frequency, $\omega_{\circ}\left(\lambda_{\circ}\right)$ is the laser frequency (wavelength), $\mathrm{v}_{\mathrm{osc}}\left(\mathrm{v}_{\mathrm{e}}\right)$ is the electron 
oscillation (thermal) velocity, and $L$ is the interaction length. For a $500 \mu \mathrm{m}$ interaction length, $\mathrm{Q}$ is on the order of 40; if the gain were this high, pump-depletion would dominate. Instead, only a small fraction of the pump energy is observed to transfer to the probe.

Many factors could lead to local detuning of the threewave resonance which in turn could limit the gain. If the plasma becomes sufficiently turbulent on a scale approaching the length of the resonant gain region, the probe might pass through the resonant flow velocity without gaining much energy. Such turbulence could take the form of velocity fluctuations produced by spatially nonuniform heater and interaction beams $[9,17,27]$. These laser "hot spots" can also modify the plasma's local heating rate and hence the electron distribution function, creating fluctuations in the ion acoustic frequency [28] which could further spatially detune the resonance condition in our experiment. Both of these effects would be enhanced by large-scale filamentation of the pump beam [29].

Numerical simulations of such plasmas with strong gradients show further reductions of the amplification. As mentioned above, the code BZOHAR has been used to perform simulations of this experiment on small spatial scales [26]. After an initial saturation, the energy transfer quickly relaxes (after $40 \mathrm{ps}$ ) and becomes nearly proportional to the input probe intensity, as seen in the experiments. BZOHAR arrives at this "linear" condition by means of nonlinear 


\section{Wharton/13}

detuning and nonlinear localization of the ion wave resonance.

V. Implications for NIF

The National Ignition Facility [1] will utilize many pairs of crossing-beams in a flowing plasma, and therefore these results may be applicable to experimental design of NIF and other indirect-drive laser fusion experiments. Numerous differences between the experimental laser and plasma parameters and those present in NIF complicate a quantitative comparison. For example, NIF plans utilize a mixed H/He gas fill inside the gold-shell "hohlraum", as opposed to the Be plasma discussed here; therefore the NIF ion waves will be more heavily damped. Specifically, the damping parameter in the Nova experiment ( Im $\omega_{\text {ia }} / \operatorname{Re} \omega_{i a}=0.1$ ) will be double for the fast-ion mode in $\mathrm{H} / \mathrm{He}$, and quadruple for the slow-ion mode (2-species ion waves are discussed in $[30,31])$. The larger damping, while possibly lowering the levels of energy transfer, will also increase the volume of the resonant region; therefore the net effect is unknown. Still, having several ion wave modes (with different sound speeds) will increase the likelihood that a resonance will occur in the NIF beam-crossing volume. For this reason, we speculate that perhaps a single-species gas fill in the hohlraum (He only) could help to detune cross-beam resonances.

NIF will have a "two-color" capability, which will allow beams at different cone-angles to have slightly different 
frequencies. If necessary, this capability can be used to detune a resonance by shifting the Mach 1 resonance out to flow velocities as high as Mach 2; a velocity which is safely outside the NIF beam-crossing volume. In a single-species plasma, this "two-color" capability should be sufficient to prevent a resonance with an ion wave.

Another difference between the NOVA experiments and NIF is the laser beams themselves; smoothing by spectral dispersion (SSD) will be present on the NIF beams. Although the addition of frequency modulation was seen to decrease the resonant energy transfer in these experiments, it should be noted that SSD would not necessarily have the same effect. If the beams are both modulated and dispersed, then the entire laser bandwidth is present at all times; instead of modulating the resonance, the region of resonant plasma might simply grow larger. For example, SSD could potentially create a resonance (say, at Mach 0.8) where no resonance existed for single-frequency beams. However, with a realistic spectral bandwidth, the two-color capability mentioned above should be sufficient to detune even this broader resonance. The use of smoothing techniques which do not increase the laser bandwidth (e.g. polarization smoothing) would further limit the potentially resonant regions.

Finally, we note that the frequency-modulation hardware used on these experiments will be available on NIF, as part of the planned SSD apparatus. If it is necessary to use a 


\section{Wharton/15}

target or laser configuration for which an energy-exchanging resonance cannot be avoided, a frequency modulation on the NIF beams may limit the energy transfer, as seen in the experiments presented above. A sufficiently fast modulation might be able to prevent a resonance from growing, even if it cannot be detuned.

VI . CONCLUSIONS

In summary, enhanced transmission of a laser beam is observed when it is crossed with a higher-intensity beam of the same frequency in a flowing plasma. Positional and temporal scaling of this effect strongly suggests that this is due to a resonance with an ion wave that has zero frequency in the laboratory frame. Frequency modulation on the crossing beams seems to decrease the resonant energy transfer by roughly a factor of two. These results may have implications for both the laser beams and the target-design in NIF and other laser-fusion schemes.

This work was performed under the Science Use of Nova program, under the auspices of the U.S. Department of Energy by Lawrence Livermore National Laboratory under contract No. W-7405-ENG-48. 
References:

[1] S.W. Haan, S.M. Pollaine, J.D. Lindl et al., Phys.

Plasmas 2, 2480 (1995).

[2] N.M. Kroll, A. Ron, and N. Rostoker, Phys. Rev. Lett. 13, $83(1964)$.

[3] V.N. Tsytovich and A.B. Shvartsburg, Sov. Phys. Tech.

Phys. 11, 1431 (1967); L.M. Kovrizhnykh, Sov. Phys. Tech.

Phys. 11, 1183 (1967); V. Stefan, B.I. Cohen, and C. Joshi, Science 243, 494 (1989).

[4] B.L. Stansfield, R. Nodwell, and J. Meyer, Phys. Rev. Lett. 26, 1219 (1971)

[5] T. Tajima and J.M. Dawson, Phys. Rev. Lett. 43, 267

[6] C.E. Clayton, C. Joshi, C. Darrow, and D. Umstadter, Phys. Rev. Lett. 54, 2342 (1985).

[7] C.E. Clayton, K.A. Marsh, A. Dyson et al., Phys. Rev. Lett. 70, 37 (1993) .

[8] C.J. Pawley, H.E. Huey, and N.C. Luhmann, Jr., Phys. Rev. Lett. 49, 877 (1982); C.W. Domier and N.C. Luhmann, Jr., Phys. Rev. Lett. 69, 3499 (1992).

[9] R.K. Kirkwood, B.B. Afeyan, W.L. Kruer et al., Phys. Rev. Lett. 76, 2065 (1996).

[10] D.M. Villeneuve, H.A. Baldis, and J.E. Bernard, Phys. Rev. Lett. 59, 1585 (1987)

[11] A.K. Lal, K.A. Marsh, C.E. Clayton, C. Joshi, C.J. McKinstrie, J.S. Li, and T.W. Johnston, Phys. Rev. Lett. 78, 670 (1997). 
[12] V.V. Eliseev, W. Rozmus, V.T. Tikhonchuk, and C.E. Capjack, Phys. Plasmas 3, 2215 (1996).

[13] C.J. McKinstrie, J.S. Li, R.E. Giacone, and H.X. Vu, Phys. Plasmas 3, 2686 (1996).

[14] R.K. Kirkwood, B.J. MacGowan, D.S. Montgomery et al., Phys. Plasmas 4, 1800 (1997).

[15] I.M. Begg and R.A. Cairns, J. Phys. D 9, 2341 (1976).

[16] R.W. Short and E.A. Williams, Phys. Rev. Lett. 47, 337 (1981); C.J. Randall, J.R. Albritton, J.J. Thomson, Phys. Fluids 24, 1474 (1981); K. Baumgärtel and K. Sauer, Phys. Rev. A 26, 3031 (1982); R.W. Short and E.A. Williams, Phys. Fluids 26, 2342 (1983).

[17] W.L. Kruer, S.C. Wilks, B.B Afeyan, and R.K. Kirkwood, Phys. Plasmas 3, 382 (1996).

[18] C.J. McKinstrie, V.A. Smalyuk, R.E. Giacone and H.X. Vu, Phys. Rev. E 55, 2044 (1997).

[19] H.A. Rose and S. Ghosal, Phys. Plasmas 5, 1461 (1998). [20] K.B. Wharton, R.K. Kirkwood, S.H. Glenzer et al., Phys. Rev. Lett. 81, 2248 (1998).

[21] S.N. Dixit, M.D. Feit, M.D. Perry, and H.T. Powell, Optics Lett. 21, 1715 (1996).

[22] G. Zimmerman and W. Kruer, Comments Plasma Phys. Controlled Fusion 2, 85 (1975).

[23] S. Glenzer, C.A. Back, K.G. Estabrook et al., Rev. Sci. Instrum. 68, 641 (1997).

[24] J.D. Moody, B.J. MacGowan, R.K. Kirkwood and D.S. Montgomery, Review of Sci. Instr. 68, 1725 (1997). 
[25] B.I. Cohen, B.F. Lasinski, A.B. Langdon, and E.A. Williams, Phys. Plasmas 4, 965 (1997).

[26] B.I. Cohen, B.F. Lasinski, A.B. Langdon, E.A. Williams, K.B. Wharton, R.K. Kirkwood, and K.G. Estabrook, Phys. Plasmas 5, 3408 (1998).

[27] B.B. Afeyan, R.L. Berger, T.B. Kaiser, and W.L. Kruer, Bull Am Phys Soc 40, 1822 (1995); D.S. Montgomery, B.B. Afeyan, J.A. Cobble et al., Phys. Plasmas 5, 1973 (1998). [28] B.B. Afeyan, A.E. Chou and W.L. Kruer, ICF Quarterly Report, LLNL (1997) and submitted to Phys. Rev. E; B.B. Afeyan et al., Phys. Rev. Lett. 80, 2032 (1998). [29] A.J. Schmitt and B.B. Afeyan, Phys. Plasmas 5, 503 (1998) .

[30] H.X. Vu, J.M. Wallace, and B. Bezzerides, Phys. Plasmas 1, 3542 (1994).

[31] E.A. Williams, R.L. Berger, R.P Drake et al., Phys. Plasmas 2, 129 (1995). 
Wharton/19

Figure Captions:

FIG. 1. (a) Thomson scattering image from a $\lambda=526.6 \mathrm{~nm}$ probe focused at $z=500 \mu m$ from the original foil position, resolved in wavelength and time. The upshifted ion acoustic wave feature overlaps the unshifted light at $t=2.9 \mathrm{~ns}$, signifying a Mach 1 plasma flow. (b) The LASNEX calculation of the Mach 1 flow location is plotted against time; $z=0$ is the original position of the foil. The measurement from Fig. 1a is shown in comparison. The dashed box represents the typical spatial and temporal extent of the crossed beams in the primary experiment.

FIG. 2. The experimental geometry is shown. The $5 \mu \mathrm{m} \mathrm{Be}$ foil is initially at $z=0$, where $z$ is the normal to the foil in the direction of the Pump beam propagation. At $t=0 \mathrm{~ns}$ two heater beams (not shown) illuminate the foil from both sides. At $t=1$ ns the Pump and Probe laser beams intersect at a known distance from the foil, at a $152^{\circ}$ angle. The diamond-shaped crossing region can have a z-extent of $850 \mu \mathrm{m}$ to $1300 \mu \mathrm{m}$ depending on the focal spot sizes, but $>75 \%$ of the intensity intersects in a region only half this size.

FIG. 3. (a) Probe transmission fraction ( $\left.T_{\text {probe }}\right)$ is plotted against time for a Pump/Probe intensity ratio of $\sim 3$. The thick solid line is the probe-only (no pump) condition. The other data show beam-crossing locations of $z=-500 \mu m$ (thin solid line) and $z=-750 \mu m$ (thin dashed line). (b) Probe 
Wharton $/ 20$

transmission fraction is plotted against time for a Pump/Probe intensity ratio of $\sim 25$. The thick solid line is the probe-only condition. The other data show beam-crossing locations of $z=-500 \mu m$ (thick dashed line), $z=0 \mu m$ (thin solid line), and $z=+500 \mu m$ (thin dashed line).

FIG 4: Probe transmission fraction ( $T_{\text {probe }}$ ) is plotted against time for a Pump/Probe intensity ratio of 25 ; 2-color bandwidth is used on both pump and probe beams. The dashed line is the probe-only (no pump) condition. The solid line shows the beam-crossing location of $z=-500 \mu m$.

FIG 5. The enhancement factor of the probe beam (Gprobe averaged from $2 \mathrm{~ns}<\mathrm{t}<3 \mathrm{~ns})$ is plotted versus position for each crossed-beam location. Circles are for Pump/Probe intensity ratios of 3 ; squares are ratios of 25 . The triangle represents a shot with frequency-modulated beams, also with a Pump/Probe intensity ratio of 25 . The large horizontal error bars represent the region over which $>80 \%$ of the crossed beams overlap. The shaded region represents the calculated Mach 1 location during the 2ns $<t<3 n s$ period. 
Figure 1

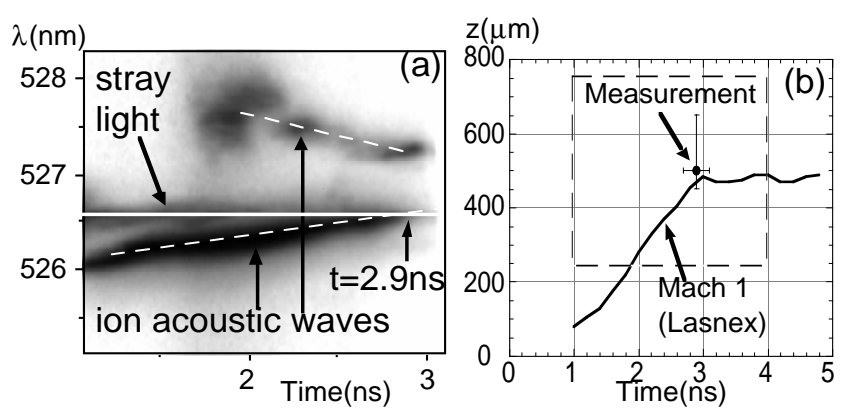


Figure 2

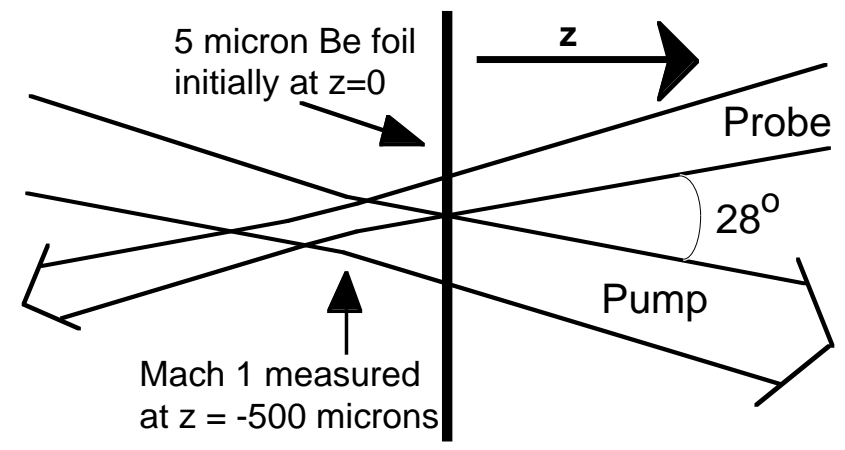


Figure 3. (Note: please make this Figure 2-columns wide)
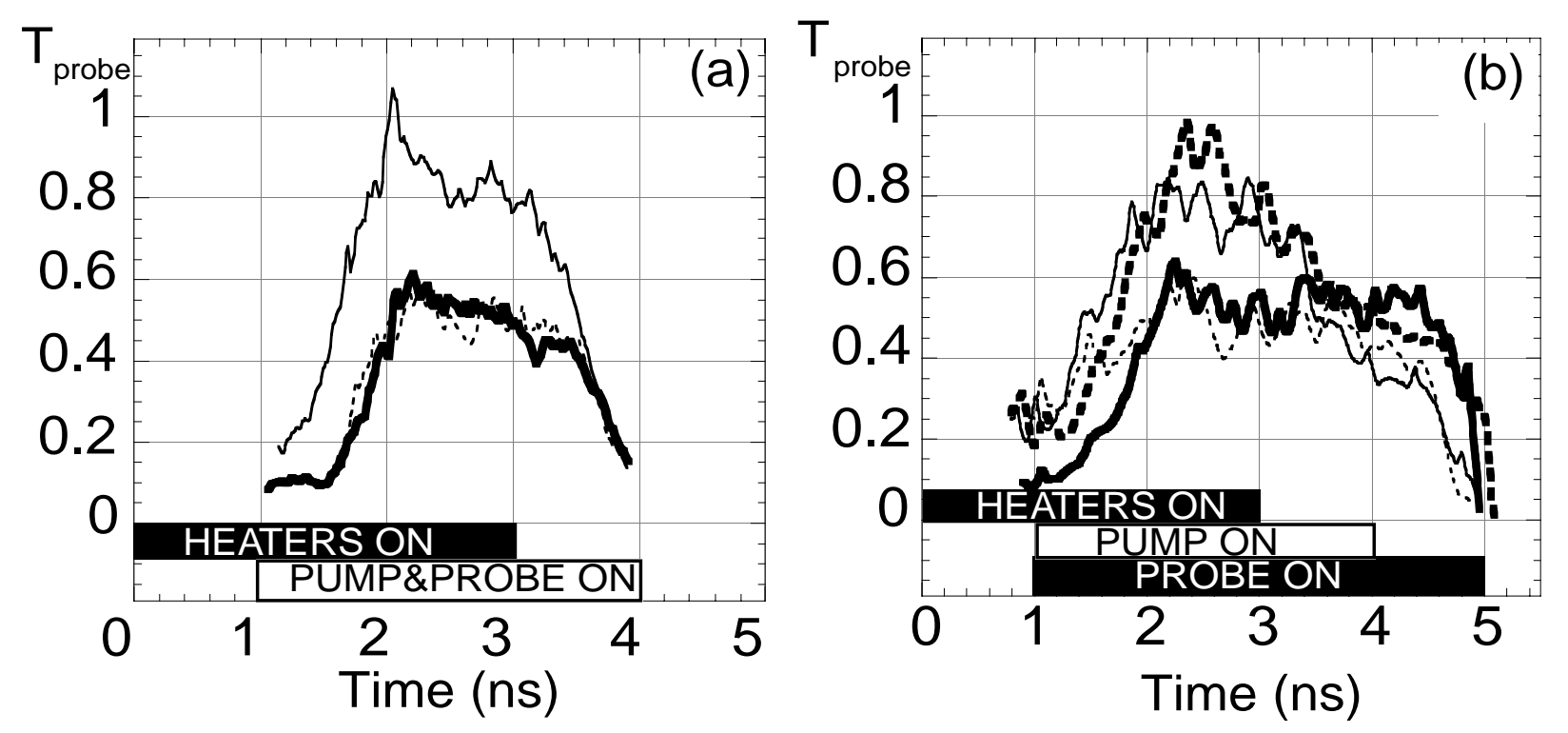
Wharton/24

Figure 4

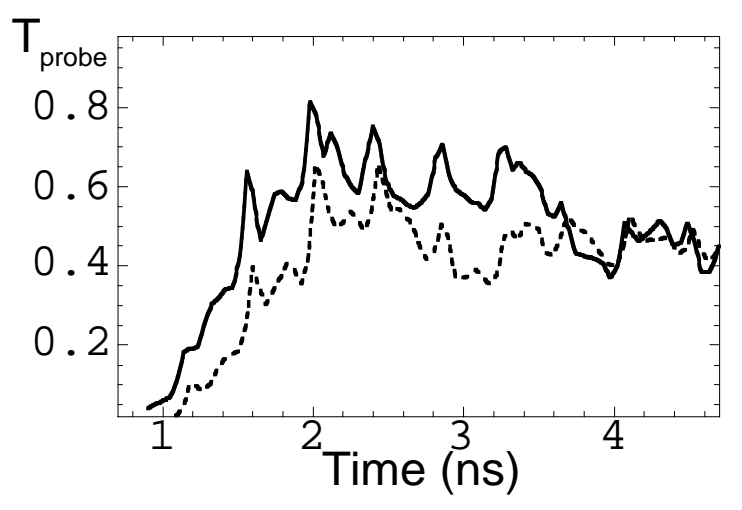


Wharton/25

Figure 5

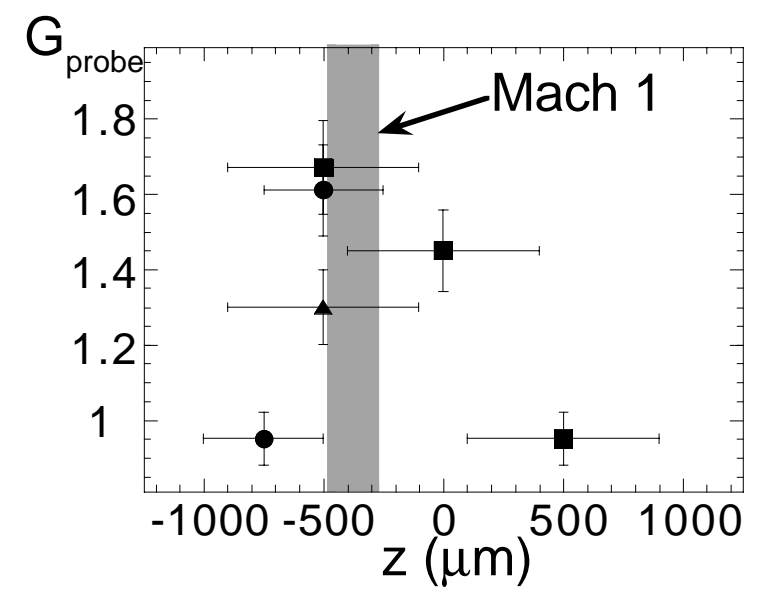

